\title{
Gold Nanoshells and Nanorings for Photo-Thermal Therapeutic Medicine
}

\section{A. Duarte-Moller}

Centro de Investigación en Materiales Avanzados (CIMAV), Laboratorio Nacional de Nanotecnología, Miguel de Cervantes No. 120, 31136 Chihuahua, Chihuahua, México.

Amongst the wide range of clinical imaging modalities available to clinicians today, advanced optical imaging systems such as optical coherence tomography (OCT), confocal reflectance endomicroscopy and diffuse optical tomography etc. offer promising clinical application particularly in the area of cancer imaging due to the high resolution they afford. However, these novel optical systems rely on scattering processes in tissue which can be rather indiscriminate in highlighting diseased states. The application of metallic nanoparticles such as gold nanoparticles as contrast agent in these reflectance-based imaging techniques is well appreciated, but limited to a narrow optical excitation range that are appropriate for use with only certain optical systems with matching optical wavelength [1-3].

The narrow optical excitation range is due to its rather invariable optical plasmon resonance, typically at $520 \mathrm{~nm}$. Although its plasmon resonance is known to vary with size, the plasmon resonance of gold nanoparticles hardly changes by more than $60 \mathrm{~nm}$ before their sizes become impractical for biological applications. Gold nanoshells have the singular property to show an absorption band around the $760 \mathrm{~nm}$, which is the same that the biological human optical window [4]. Here we discuss the production of gold nanoshells and the formation of gold nanorings by using of $\mathrm{SiO}_{2}$ nanosphere template.

Hydrogen tetrachloroaurate $\left(\mathrm{HAuCl}_{4} \cdot 3 \mathrm{H}_{2} \mathrm{O}, 99.99 \%\right)$, 3-aminopropyltrimethoxysilane (APTMS, 95\%) and tetraethyl orthosilicate (TEOS, 98\%) were purchased from Sigma (USA), Triton X-100 (TX-100) was purchased from Aldrich, cyclohexane, n-hexanol, trisodium citrate, sodium brohydride $\left(\mathrm{NaBH}_{4}\right.$, $98 \%)$, ammonia water $\left(\mathrm{NH}_{4} \mathrm{OH}, 25 \%\right)$, absolute ethanol and polyethylenimine (PEI) with average molecular weight $600,000 \mathrm{~g}$ mol-1 were obtained from Beijing. All reagents used were analytical grade and without further purification. The colloidal $\mathrm{Au}$ was prepared by rapidly adding $1.2 \mathrm{~mL}$ of $1 \%$ trisodium citrate to $100 \mathrm{~mL}$ of $0.01 \% \mathrm{HAuCl}_{4}$ aqueous solution when $\mathrm{HAuCl}_{4}$ solution got a rolling boil under vigorous stirring. The color changed from pale to blue, then turned to burgundy. Boiling was continued for $15 \mathrm{~min}$, stirring until it reached room temperature.

The method to obtain gold nanoshells by using the $\mathrm{SiO}_{2}$ templates was very easy. Functionalized gold nanoaparticles were successfully assembled in the $\mathrm{SiO}_{2}$ surface. The absorption wavelength was that required (NIR) for the optical human window.

$\mathrm{Au} / \mathrm{SiO}_{2}$ nanoshells can be converted to nanorings upon addition of excess $\mathrm{KAuCl}_{4}$. Nanorings present a distinct particle geometry, with optical properties exhibiting characteristics of both nanorods and nanoshells. The gold nanoparticles, which are in the size range 4-5 nm, are used due that have a plasmon resonance with incoming radiation causing them to both absorb and scatter light. This effect can be harnessed to either destroy tissue by local heating or release payload molecules of therapeutic importance. Gold nanoparticles can also be conjugated to biologically active moieties, providing possibilities for targeting to particular tissues. 


\section{References:}

[1] R Bhattacharya and P Mukherjee, Adv. Drug Delivery Rev. 60 (2008) p. 1289.

[2] P K Jain, X Huang, I H El-Sayed and M A El-Sayed 41 (2008) p. 1578.

[3] P C Chen, S C Mwakwari and A K Oyelere, Nanotechnol., Sci. Appl. 1 (2008) p. 45.

[4] V P Zharov, K E Mercer, E N Galitovskaya and M S Smeltzer, Biophys. J. 90 (2006) p. 619.

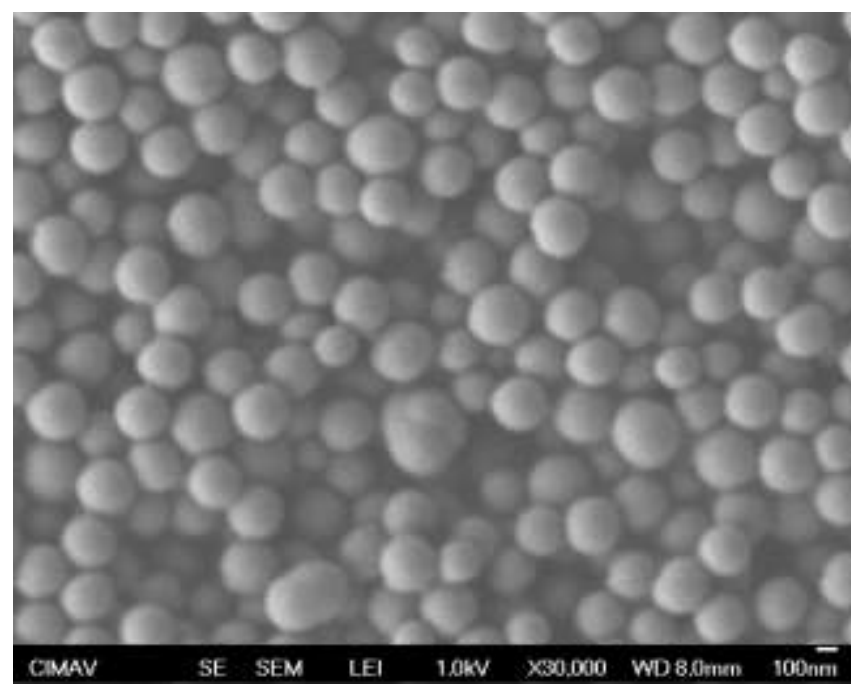

Figure 1. FESEM-SE micrograph of synthesized $\mathrm{SiO}_{2}$ nanospheres. High uniformity can be observed among the particles.
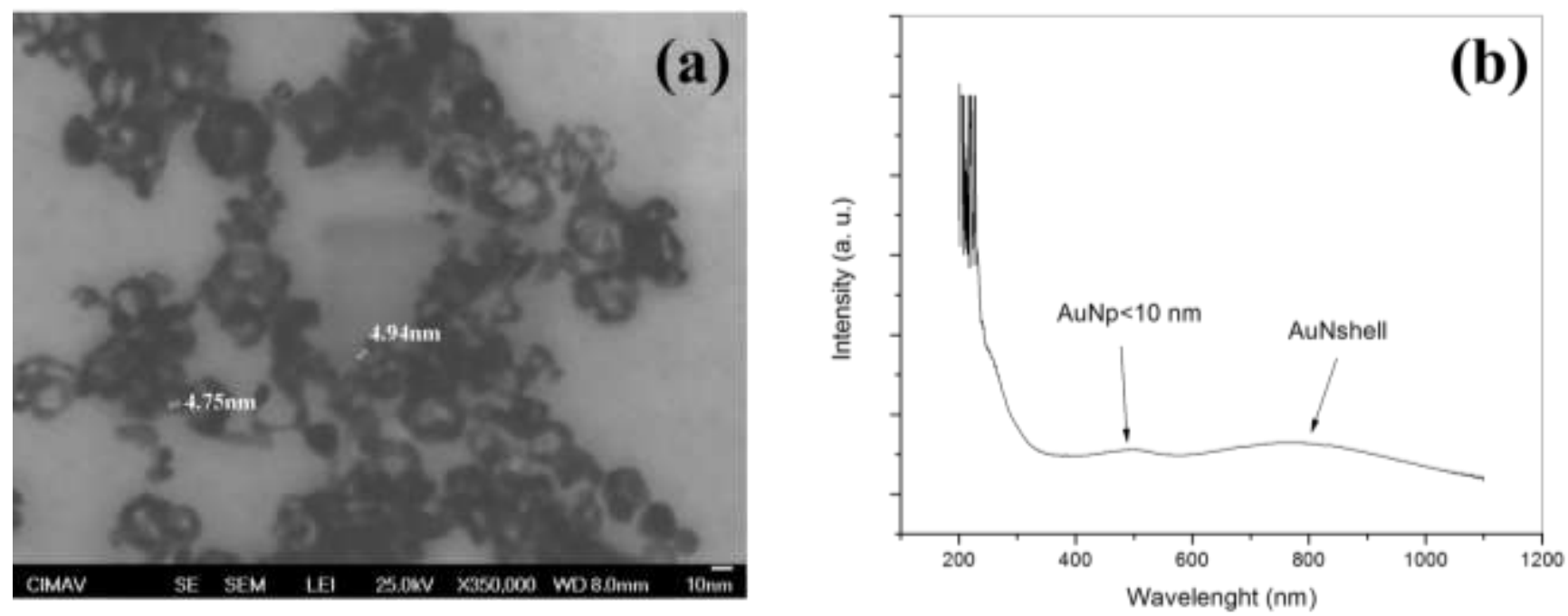

Figure 2. (a) FESEM-SE micrograph of the gold nanoshells with different shapes and sizes, including those nanorings formed in the experimental procedure. (b) Absorption spectra, when there is a border around the $500 \mathrm{~nm}$, corresponding to the gold nanoparticles band and a band placed at $770 \mathrm{~nm}$. This band is associated with that corresponding to the gold nanoshell. 